\title{
Installation and Docking Technology of Control Module for Subsea Production System
}

\author{
Lan Zhang ${ }^{\mathrm{a}}$, Yongzhe $\mathrm{Li}^{\mathrm{b}}$ Yonghong Meng $^{\mathrm{c}}$, Liquan Wang ${ }^{\mathrm{d}}$, Peng Jia ${ }^{\mathrm{e}}$ \\ School of Mechanical and Electrical Engineering, Harbin Engineering University, Harbin 150001, \\ China. \\ azhanglan@hrbeu.edu.cn, b1360587603@qq.com, c1634919507@qq.com, \\ dwangliquan@hrbeu.edu.cn, ejiapeng@hrbeu.edu.cn
}

\begin{abstract}
Keywords: Subsea control module, running tool, subsea docking, positional accuracy, flow analysis, pressure drag, running and retrieval, water-exit speed.
\end{abstract}

\begin{abstract}
The docking technology of the subsea production system control module (SCM) is a key part of installation. If the docking accuracy is not reached, or poor stability when docking, the butt joint between the hydraulic joint and the electrical joint cannot be accomplished, may also make the subsea control module not work. Therefore, aiming at the design and installation requirements of SCM for 1500 meters of water depth, it focuses on the research of subsea installation and stable retracting technology. Based on the computational fluid mechanics method, the simulation of the flow field and the analysis of the effluent multiphase flow in the deployment and reclamation process are carried out, and the theoretical basis is provided for the hydrodynamic characteristics and the control of the effluent stability of the equipment in the deployment and reclamation process. The results show that the coupling between SCM and MRT can be applied to the positioning and docking, and the positioning accuracy can reach $0.1 \mathrm{~mm}$, which increases $44 \%$ compared with other methods. When deploying, the differential pressure between SCM and MRT is 4 times, the cable tension required is larger, and the stability is poor than the alone installation tool. When recycling, with the increase in reclamation speed, the device's pressure drag gradually increased, the friction drag changes slightly, and the overturning moment of the equipment in the water-exit process decreases as the water-exit speed increases. In this way, the designed docking device can meet the installation requirements, and ensure the accuracy and stability of the docking.
\end{abstract}

\section{Introduction}

Subsea Control Module (abbreviated as SCM) is used to control the valve on X-mas tree, manifold and separator in the subsea production system and is a module device collecting the data in the subsea production facilities to conduct dispose and feedback [1]. The transmission information between SCM and subsea production facilities is transmitted through the electro hydraulic connector on the SCM docking plate.

At present, the SCM deployment and docking mainly rely on the module running tool (abbreviated as MRT) to achieve, and MRT is a kind of marine engineering equipment which can perform subsea control module deployment, buffering, docking and reclamation tasks[2]. This device has rarely been studied in China, and the deployment and docking technology is always the core technology of subsea control module installation. Foreign research on MRT started from the birth of SCM, but the domestic research on marine subsea control module is still in its infancy and limited to theory[3]. The docking of subsea production system control module (SCM) is the key link of its installation, which directly affects the accurate butt joint of electro hydraulic joints. When the docking accuracy is not reached, or when the stability is poor, the subsea control module fails, and it will be difficult to complete the specific work.

\section{The Analysis on Docking and Locating Between SCM and MRT}

The installation docking of subsea control module is mainly through the docking plate and the docking device on MRT to complete, and mainly consist of four parts: the sliding key on MRT and 
the chute on the protection bucket; locating pin on the upper docking plate and the flare opening on the lower docking plate; the cone on the upper docking plate and the guide sleeve on the lower docking plate; the guide groove on the upper docking plate and the locating key on the lower docking plate, whose positioning structure is shown in Figure 1.

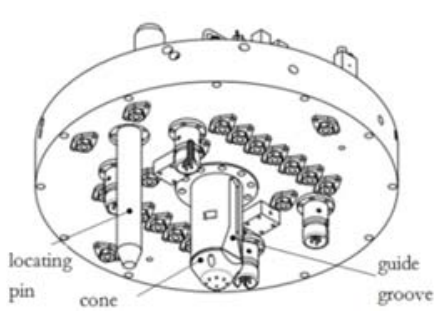

(a)The upper docking plate

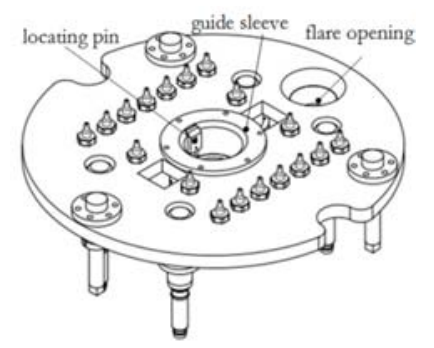

(b) the lower docking plate

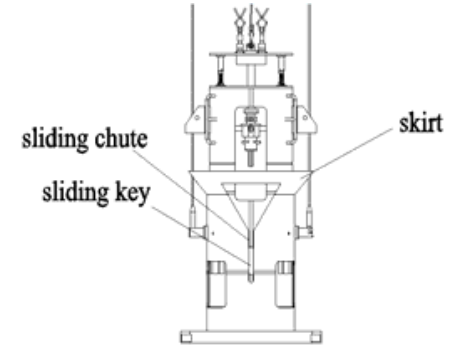

(c) MRT and mounting base

Fig. 1 Positioning structure

When install SCM in the deep water, there are many uncertain factors, such as the sea wind and waves, ocean currents and other factors, meanwhile, ROV operating capacity is limited, which cannot directly complete the precise docking, so positional accuracy cannot be too high in the initial docking process, however, it is a process from coarse to fine, step by step. Specific positioning process of SCM installation system is as follows:

1)the shell of MRT enter into the skirt

In the docking process, the radial clearance should be reasonable between SCM and MRT, otherwise, it affect the docking accuracy or is stuck. According to the characteristic of the MRT guide structure, in the process of installation,

$$
x=a-c
$$

In the formula, $\mathrm{x}$ - the maximum value of radial redundancy, $\mathrm{mm}$;

a- the maximum capture diameter of skirt, designed value is $1600 \mathrm{~mm}$;

$\mathrm{b}$ - the minimum capture diameter of skirt; designed value is $1090 \mathrm{~mm}$;

$\mathrm{c}$ - external diameter of installation, designed value is $1060 \mathrm{~mm}$.

$$
y=b-c
$$

In the formula, $\mathrm{y}$ - the minimum value of radial redundancy, $\mathrm{mm}$;

Under the influence of the ocean currents and other factors, MRT may produce the tilt angle, thus producing the minimum gap in the docking process,

$$
\Delta=f(b, c)=b-\frac{c}{\cos \alpha^{\circ}}
$$

In the formula, $\Delta$ - the minimum gap, mm;

$\alpha$ - the tilt angle ${ }^{\circ}$.

The shell of MRT enters the skirt in the maximum inclination angle $\alpha=6^{\circ}$, and calculated by the formula (3), achieve the minimum gap is $24 \mathrm{~mm}$, making the MRT be successfully inserted into the installation base, as is shown in Figure 2.

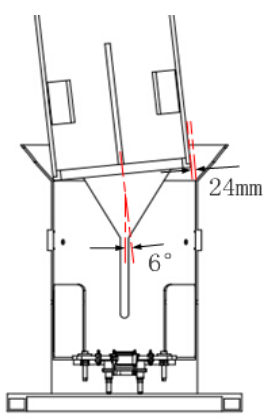

Fig. 2 The docking process at the tilt angle $6^{\circ}$

2) Sliding key enter into the chute 
When the sliding key enters into the funnel-shaped chute, the MRT is rotated in the axial direction until the sliding key enters the vertical chute, which limits the drilling degree of freedom around the z-axis, but MRT can slide up and down at the z-axis direction, meanwhile on account of the existence of the gap, MRT can be moved in the y-axis direction, and there are two extreme positions, whose the limit radial distance is $30 \mathrm{~mm}$, so MRT can be adjusted in this range along the y-axis direction. No matter where the limit position is, the cone on the upper docking plate and the top of the locating pin can enter the guide sleeve and flare opening, as is shown in Fig 3.

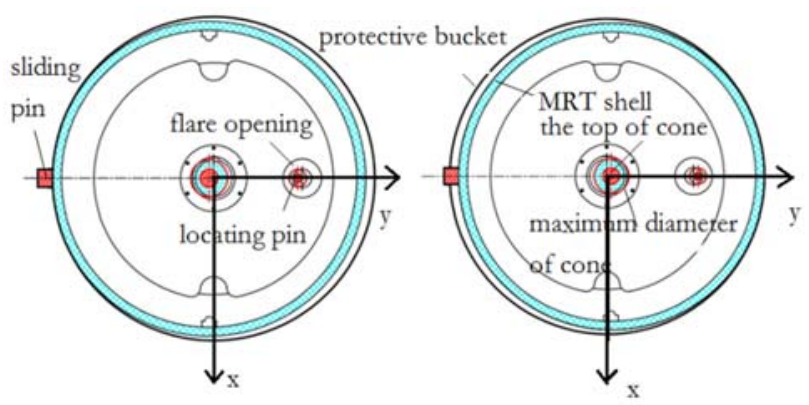

Fig. 3 MRT radial limit position docking diagram

3) Locating pin enter into the flare opening

When installing with MRT, the length of the locating pin is greater than the guide cone, so that the position can be adjusted with the locating pin when the MRT has not yet come into contact with the bottom of the barrel. When the locating pin enters the flare opening, whose inlet diameter is $137 \mathrm{~mm}$, and the outlet diameter is $62 \mathrm{~mm}, \mathrm{SCM}$ slides down along the bevel slope, and gradually reduced the radial deviation. The locating pin diameter is $60 \mathrm{~mm}$, regardless of any limit position, the radial distance will eventually be reduced to less than $2 \mathrm{~mm}$.

4) Cone enter into the guide sleeve

The guide key moves along the guide groove, defining the SCM rotation, and when the cone enters the guide sleeve, the maximum diameter of the cone is $125 \mathrm{~mm}$, and tolerance value is $\mathrm{h} 9$, whose upper deviation is $0 \mathrm{~mm}$, and whose lower deviation is $-0.1 \mathrm{~mm}$, while the mating diameter of guide sleeve is $125 \mathrm{~mm}$, and tolerance value is $\mathrm{H} 9$, whose upper deviation is $+0.1 \mathrm{~mm}$, and whose lower deviation is $0 \mathrm{~mm}$. When the technology reaches the limit position of the tolerance requirement, the center deviation distance is $0.05 \mathrm{~mm}$, that is, the center deviation distance is less than $0.05 \mathrm{~mm}$, the clearance distance is less than $0.1 \mathrm{~mm}$, and the maximum gap is reduced to $0.1 \mathrm{~mm}$. When the center deviation of the male and female coupling is less than $0.54 \mathrm{~mm}$, it can be precisely docked [4], so achieve the precise docking of electric hydraulic joint. When the bottom of the shell of the MRT contact with the bottom of the protective bucket, the entire dynamic positioning process is completed. According to the relevant information, in the design on subsea control module docking mechanism, the maximum central deviation between the key and keyway is $0.09 \mathrm{~mm}$. Compared with it, the docking accuracy designed increased by $44 \%$.

The above process is through the horn-shaped device gradually adjusted until the last precise positioning, then docking transition automatically is completed. As the Electric hydraulic joints are more on the docking plate, no matter how accurate MRT docking position is, cannot eliminate the influence on machining error for the dock and electro-hydraulic couplings. Multiple joints are difficult to plug simultaneously and the correct docking, and there are no stress concentration and sealing problems, so the male hydraulic coupling on the lower docking plate install with a floating installation method. That is, the diameter of the mounting hole is greater than the diameter of the hydraulic couplings mounting surface $0.1 \mathrm{~mm}$, at the moment, 12-way hydraulic connector and 12way electrical connector can be docked accurately.

\section{Flow Field Analysis on the Deployment and Reclamation Process of SCM and MRT}

For operation characteristics on operating condition of subsea control module, in order to obtain the hydrodynamic characteristics of the equipment and define the main factors influencing the 
installation, the model pressure and velocity data are extracted, and the equations are calculated based on the Renault average NS equations (RANS). The control equation is[5],

$$
\left\{\begin{array}{l}
\frac{\partial U_{i}}{\partial x_{j}}=0 \\
\frac{\partial U_{i}}{\partial t}+\frac{\partial}{x_{j}}\left(U_{i} U_{j}\right)=\frac{1}{\rho} \frac{\partial P}{\partial x_{j}}\left(\Gamma_{i j}-\rho \overline{u_{i}} \bar{u}_{j}^{\prime}\right)
\end{array}\right.
$$

For solving the turbulence model of the N-S equation, the achievable $k-\varepsilon$ model is usually used in the engineering process:

$$
\left\{\begin{array}{l}
\frac{\partial(\rho k)}{\partial t}+\frac{\partial\left(\rho k u_{i}\right)}{\partial x_{i}}=\frac{\partial}{\partial x_{j}}\left[\left(\mu+\frac{\mu_{t}}{\sigma_{k}}\right) \frac{\partial k}{\partial x_{j}}\right]+G_{k}+G_{b}-\rho \varepsilon-Y_{M} \\
\frac{\partial(\rho \varepsilon)}{\partial t}+\frac{\partial\left(\rho \varepsilon u_{i}\right)}{\partial x_{i}}=\frac{\partial}{\partial x_{j}}\left[\left(\mu+\frac{\mu_{t}}{\sigma_{\varepsilon}}\right) \frac{\partial \varepsilon}{\partial x_{j}}\right]+\rho C_{1} E \varepsilon-\rho C_{2} \rho \frac{\varepsilon^{2}}{k+\sqrt{v \varepsilon}}+C_{1 \varepsilon} \frac{\varepsilon}{k} C_{3 \varepsilon} G_{b}
\end{array}\right.
$$

Two states of MRT deployment and reclamation consist of four processes, which are MRT alone deployment and reclamation, another are simultaneous deployment and reclamation of SCM and MRT. Two-dimensional flow field analysis model is established in the pretreatment software Pointwise and the meshing is completed.

The installation of the subsea production facility has a certain speed requirement. Running speed of subsea control module and ROV laying reclamation system are generally controlled at about 0.4 $\mathrm{m} / \mathrm{s}$ [6], which determines the stability of the running tool and the installed equipment. Different equipment has different water resistance due to its own structure, according to the literature [7], select the speed of $0.4 \mathrm{~m} / \mathrm{s}$ to mock up subsea movement process in the Fluent of software, as is shown in Figure 4.
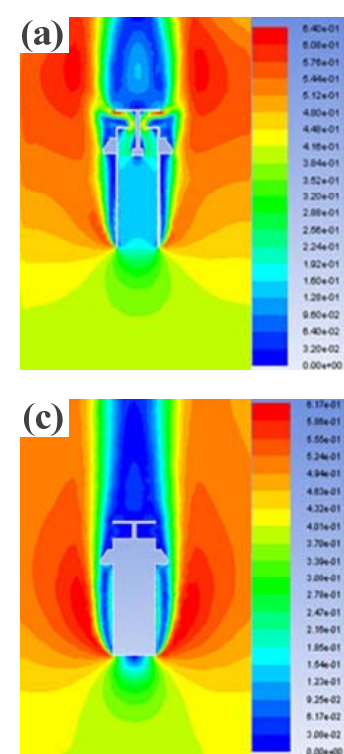

(b)

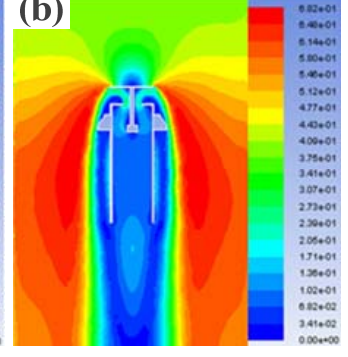

(d)

Fig. 4 Distribution encompassing the flow field

MRT alone deployment and reclamation are shown in Fig. 7 (a) (b), forming the shape of eddy high pressure area outside of the shell, and the fluid passes from the inside of the shell, causing an impact on the edge of the upper support plate. Simultaneous deployment and reclamation of SCM and MRT are shown in Fig. 7 (c) (d). Due to the shell of MRT filled with SCM, the fluid almost cannot pass through the MRT, accompanied by a long wake. The overall movement resistance is significantly increased related to the first two states, and the overall deployment process resistance is four times than MRT alone deployment. While the resistance in overall rise process is slightly larger than the individual rise process, because the two states are similar in structure during the rise. 
By analysis, obtained that the resistance of simultaneous reclamation of SCM and MRT is the biggest, whose area is concentrated on the upper support plate, and the frictional resistance is mainly concentrated around the casing of the MRT. The same method is used to change the retracting speed from $0.3 \mathrm{~m} / \mathrm{s}-0.9 \mathrm{~m} / \mathrm{s}$, and obtain the resistance values shown in Table 1.

Through the data analysis, it can be seen that the pressure resistance of MRT increases linearly with the increase of the speed of reclamation process, but the change of frictional resistance is not too much, almost kept at $22 \mathrm{~N}$ or so. The increase of resistance will directly increase the cable tension, and because the operating depth become larger, cable drag force in addition to the gravity of the hanging material has its own gravity.

Table 1 MRT resistance on reclamation process

\begin{tabular}{|c|c|c|c|}
\hline velocity $v /(\mathrm{m} / \mathrm{s})$ & frictional resistance $F_{f} / \mathrm{N}$ & Pressure drag $F_{p} / \mathrm{N}$ & $\begin{array}{c}\text { Total resistance } \\
/ \mathrm{N}\end{array}$ \\
\hline 0.9 & 23.71 & 822.607 & 846.317 \\
\hline 0.8 & 23.62 & 731.206 & 754.826 \\
\hline 0.7 & 22.34 & 639.806 & 662.146 \\
\hline 0.6 & 21.38 & 548.405 & 569.785 \\
\hline 0.5 & 21.25 & 457.004 & 478.254 \\
\hline 0.4 & 20.17 & 365.603 & 385.773 \\
\hline 0.3 & 20.05 & 274.202 & 294.252 \\
\hline
\end{tabular}

Strongly interference by the waves near the surface of the water, meanwhile the free sea surface tension and the impact of the air, MRT will be subject to non-linear force, whose environment is quite complex [8]. If cannot be better controlled at this stage, MRT will be seriously damaged. Due to the large difference between the density and viscosity of liquid and gas, the inertial force of the effluent device at the interface reaches a change of 800 times [9], and a large torque is generated, which makes it easy to offset and overturn, especially generate a great load on the lifting mechanism of MRT.

In view of the equipment operation process in the sea conditions of the complex water surface, the gas-liquid two-phase flow field analysis is carried out with the MRT normal water flow rate of 0.4 $\mathrm{m} / \mathrm{s}$, and the waves in the four-level sea conditions are simulated in the water surface, obtaining gasliquid two-phase flow velocity nephogram of the MRT at different times before and after the effluent MRT, as shown in Figure 5.
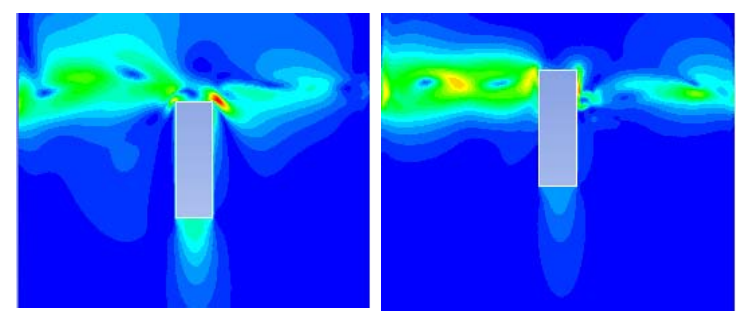

Fig. 5 Flow field analysis in the process of effluent

When MRT approach to the water surface, it has a large wake, and the velocity is larger at the area of bottom center and the top edge. When pass through the water surface, on the influence of wave and different characteristics of fluid on gas-liquid two-phase, a vortex is formed in the side of MRT, and an asymmetric flow field is formed at the upper and lower corners, producing a overturning moment. Change the water velocity to get the overturning moment of the MRT, as shown in Table 2 . The analysis shows that the overturning moment decreases as the effluent velocity increases.

Table 2 Overturning moment of the MRT in the process of effluent

\begin{tabular}{cccc}
\hline velocity $v /(\mathrm{m} / \mathrm{s})$ & torque $\mathrm{M} / \mathrm{Nm}$ & velocity $v /(\mathrm{m} / \mathrm{s})$ & torque $\mathrm{M} / \mathrm{Nm}$ \\
\hline 0.9 & 80.23 & 0.5 & 102.53 \\
0.8 & 90.25 & 0.4 & 110.87 \\
0.7 & 92.75 & 0.3 & 125.36 \\
0.6 & 100.23 & 0.2 & 128.54 \\
\hline
\end{tabular}


In order to balance the equipment high tension on cable in the high-speed and high overturning moment in low-speed reclamation, the speed must be controlled at a reasonable figure, which is around $0.5 \mathrm{~m} / \mathrm{s}$.

\section{Conclusion}

Aiming at the marine environment with a depth of $1500 \mathrm{~m}$, an automatic locating running tool for SCM is designed. According to the positioning and docking between the subsea control module and the module running tool, the subsea docking precision reached $0.1 \mathrm{~mm}$, overcoming the problem of the multiple joints plugging simultaneously, achieving precision docking between the SCM docking plates.

The turbulence model is established, and the flow field of the device is simulated and analyzed. The following conclusions are drawn:

(1) The pressure drag of simultaneous deployment of SCM and MRT is 4 times than MRT alone deployment, while the resistance of simultaneous reclamation of SCM and MRT is slightly larger than MRT alone reclamation.

(2) MRT pressure drag increases with the reclamation speed increase, while the frictional resistance does not change much;

(3) On the influence on the wave and gas-liquid two-phase flow in the MRT and SCM effluent process, the equipment overturning moment reduces with the increasing speed.

Therefore, in order to balance the equipment high tension on cable in the high-speed and high overturning moment in low-speed reclamation, the retraction speed is controlled at about $0.5 \mathrm{~m} / \mathrm{s}$, which ensures the stability of the docking and provides a reference for the further study of the engineering running tool.

\section{Acknowledgments}

The ocean engineering equipment scientific research project of Ministry of Industry and Information Technology " the development of subsea control system flange and retaining mechanism "; The ocean engineering equipment scientific research project of Ministry of Industry and Information Technology " research and development of subsea control system and key equipment" subtask " research and development of electrohydraulic composite and all electric subsea control module (SCM) principle prototype" (No.Z16SJENK0033).

\section{References}

[1]. Broadbent PA. Controls reliability and early life of field failure of subsea control modules[C]. Subsea Control and Data Acquisition (SCADA) Conference, Newcastle: One Petro, 2010: 1-8.

[2]. Erlend R. Moonpool operations on Havila subsea improvement study [D]. Norway: Faculty of Science and Technology, University of Stavanger, 2012, 7-9.

[3]. Zhang Pengju, Liang Bin, Liu Kang, Guo Jun. Research on the design of subsea control module [J]. Development \& Innovation of Machinery \& Electrical Products, 2014, 27(6):113-115.

[4]. Liu Peilin, Cao Xuewei, Su Feng, Wu Xiaoshuang, Zhou Kai, Wang Liquan. Design and docking tests of a docking mechanism for subsea control modules [J]. China offshore oil and gas, 2016, 28(5): 110-114.

[5]. Wu Nailong, Wu Chao, Ge Tong, Wang Tao, Zhang Ya. Flow Recognition of underwater vehicle based on the perception mechanism of lateral line [J]. Journal of Mechanical Engineering, 2016, 52(13): 54-59. 
[6]. Jin Yongping, Wan Buyan, Liu Deshun, Peng Youduo. Analyses of launch \& recovery system of seafloor drill under different launching and recovering velocities[J]. China Mechanical Engineering, 2015, 26(19): 2557-2563.

[7]. Fang Zifan, Xiang Bingfei, Xiao Huapan, He Kongde, Liu Junfei. Dynamic and control strategy of launch and recovery device in Harsh Sea conditions [J]. Journal of Mechanical Engineering, 2013, (15): 88-95.

[8]. Sarkar A, Gudmestad OT. Splash zone lifting analysis of subsea structures[C]. Proceedings of the ASME 2010 29th International Conference on Ocean, Offshore and Arctic Engineering, Shanghai: ASME, 2010: 1-10.

[9]. Zheng Bangtao. Overview on hydroballistics and fluid dynamics of submarine-based missiles [J]. Missiles and space vehicles, 2010, 05:8-11+55. 